\title{
Effect of radiant heat on head temperature gradient in term infants
}

Neonatal Intensive

Care Unit,

Department of

Paediatrics, National

Women's Hospital,

Auckland School of

Medicine, University

of Auckland, Private

Bag, Auckland, New

Zealand

A J Gunn

T R Gunn

Correspondence to Associate Professor Tania R Gunn.

Accepted 16 January 1996

\author{
Alistair J Gunn, Tania R Gunn
}

\begin{abstract}
Aims-To test the hypothesis that external radiant heating might lead to significant fluctuations in superficial and core head temperatures in newborn infants.

Methods-In an observation group of 14 term infants nursed under a radiant heater, servo-controlled to the abdominal skin, changes in rectal, core head, and scalp temperatures with heater activation were examined. In a further intervention group of six infants the effect of a reflective head shield on the fluctuations of scalp temperature was also tested.

Results-In the observation group, when the heater had been off for $\mathbf{3 0}$ minutes, the rectal and scalp temperatures were 36.7 (SD 0.6) and $35.6(0.6)^{\circ} \mathrm{C}$, respectively, a difference of $1.2(0.2)^{\circ} \mathrm{C}$. After 30 minutes with the radiant heater on this fell to 0.2 $(0.5)^{\circ} \mathrm{C}$. The core head temperature, however, remained similar to the rectal temperature throughout. In the intervention group a reflective shield prevented the loss of the rectal-scalp gradient.

Conclusion-Overhead heater activation is associated with loss of the core to scalp temperature gradient, but no change in core head temperature in term infants. The clincal relevance of this superficial heating in vulnerable infants warrants further study.

(Arch Dis Child 1996; 74: F200-F203)
\end{abstract}

Keywords: term; hyperthermia; radiant heaters; core head temperatures.

Recent data consistently suggest that cerebral temperature critically modulates cerebral damage after hypoxic-ischaemic insults. ${ }^{1}$ Even mild cerebral hyperthermia during or following the insult may exacerbate damage. ${ }^{23}$ Thus factors which determine head temperature in newborn infants must be examined. The brain is metabolically highly active, and produces about $70 \%$ of total body heat in the newborn. ${ }^{4}$ The heat produced is dispersed mainly by radiative loss from the scalp, and by conductive loss via the cerebral circulation. In both adult neurosurgical patients ${ }^{5}$ and in newborn infants ${ }^{7}$ the core intracerebral temperature, most closely reflected by intraventricular temperature, is reported to be higher than both body temperature (rectal or oesophageal) and the temperature of the epidural space, and its overlying skin. These gradients clearly reflect the two mechanisms of heat loss.

Thus the balance of cerebral metabolism (heat production), cerebral blood flow, and scalp radiation determines cerebral temperature. The effects of external heating of the head in term infants nursed under overhead heaters have not been studied; such infants may have been exposed to perinatal asphyxia. As radiative heat loss from the scalp is primarily determined by skin and environmental temperature,$^{89}$ external heating could cause a loss of the core to skin temperature gradient in the head. If cerebral blood flow did not increase in compensation, there might also be a rise in core head temperature.

A key advance in the study of infant temperature regulation has been the application of the 'zero gradient' method to measure core body ${ }^{10}$ and head temperatures, ${ }^{7}$ using thermistors placed between the skin and underlying mattress. This approach takes advantage of the fact that infant mattresses are very highly insulating, while warm skin conducts heat rapidly. ${ }^{10}$ Thus the skin against the mattress rapidly warms up to the core temperature, and heat flux from core to environment falls to negligible levels. Once equilibration has occurred, temperatures at such a site do not lag significantly behind changes in rectal temperature. ${ }^{7} 10$ Hence temperature recordings from sites beneath the head and body can be taken as equivalent to the core head and body temperatures.

In this study we examined sequential changes in rectal, scalp, and core temperatures in term infants for up to 48 hours after birth. As these data suggested a very rapid increase in the temperature of the scalp over the fontanelle, compared with the core body and core head temperatures, when the heater restarted after being spontaneously off for 30 minutes or more. We then compared the effect of external heating with and without a reflecting heat shield over the head of the infant, on scalp and rectal temperatures.

\section{Methods}

The sample population consisted of term infants requiring mechanical ventilation in the neonatal intensive care unit (NICU) at the National Women's Hospital. The air temperature in the NICU is maintained at $28^{\circ} \mathrm{C}$. All infants were nursed under radiant heaters servo-controlled to the abdominal skin temperature to a range of 36.5 to $37 \cdot 0^{\circ} \mathrm{C}$; Heathdyne IW10 and Air Shield (USA) models IICS-90 or SC-78 were used. Simultaneous rectal, scalp over the fontanelle, and back of head and back of trunk (at mid thorax) temperatures were measured using an eight channel, microprocessor controlled transducer unit sensitive to $0.01^{\circ} \mathrm{C},{ }^{11}$ and printed every minute; the 


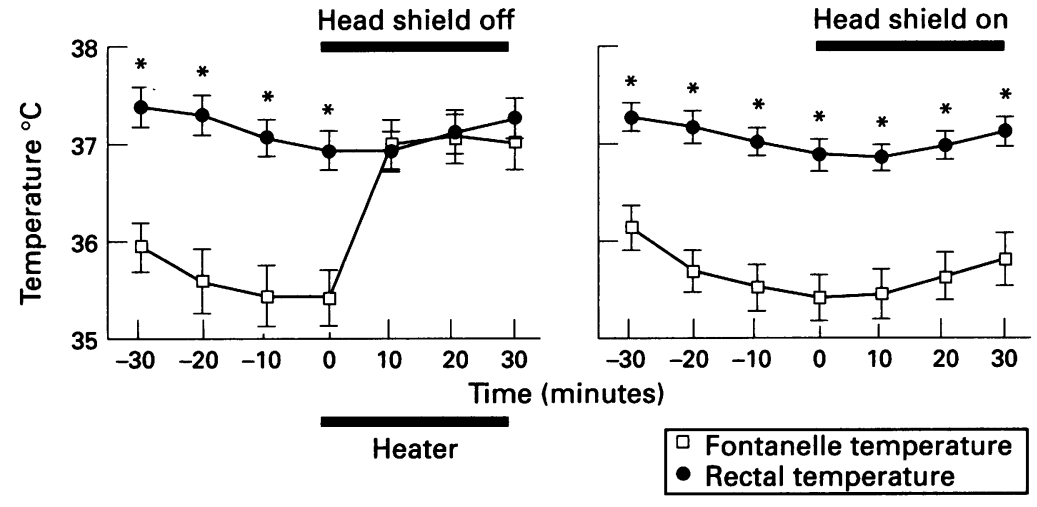

Time sequence of temperature changes in six term infants nursed under an overhead heater with and without a heat shield over the head of the infant. With the heater turned off, the temperature of the scalp over the fontanelle is significantly lower than the rectal

temperature. The heat shield maintains the normal rectal-scalp gradient when the heater is turned on $(P<0.0001)$. The temperatures are shown as mean $(S E M) .{ }^{\star} P<0.01$ rectal vs scalp temperature. temperatures were analysed every 10 minutes. A further thermistor was placed on the mattress beside the infant to document functioning of the heater. After parting the hair (on the scalp), and cleaning the skin with alcohol, the thermistors were attached by collodion glue, then covered by an insulated reflecting pad. The back of body and head thermistors (measuring, respectively, core body and head temperatures) were placed so that they were between the skin and the mattress, and were allowed fully to equilibrate before recordings were begun. The rectal thermistors were placed at $5 \mathrm{~cm}$.

\section{OBSERVATIONAL GROUP}

Two separate groups were studied. In the first observational group of 14 infants, temperatures were constantly monitored for up to 48 hours. Over this time at least one period was identified in each infant in which the overhead heater spontaneously turned off, because the skin temperature was equal to, or exceeded, the set temperature, for at least 30 minutes and then restarted; the first such episode in each

Reasons for ventilation in observation and intervention groups

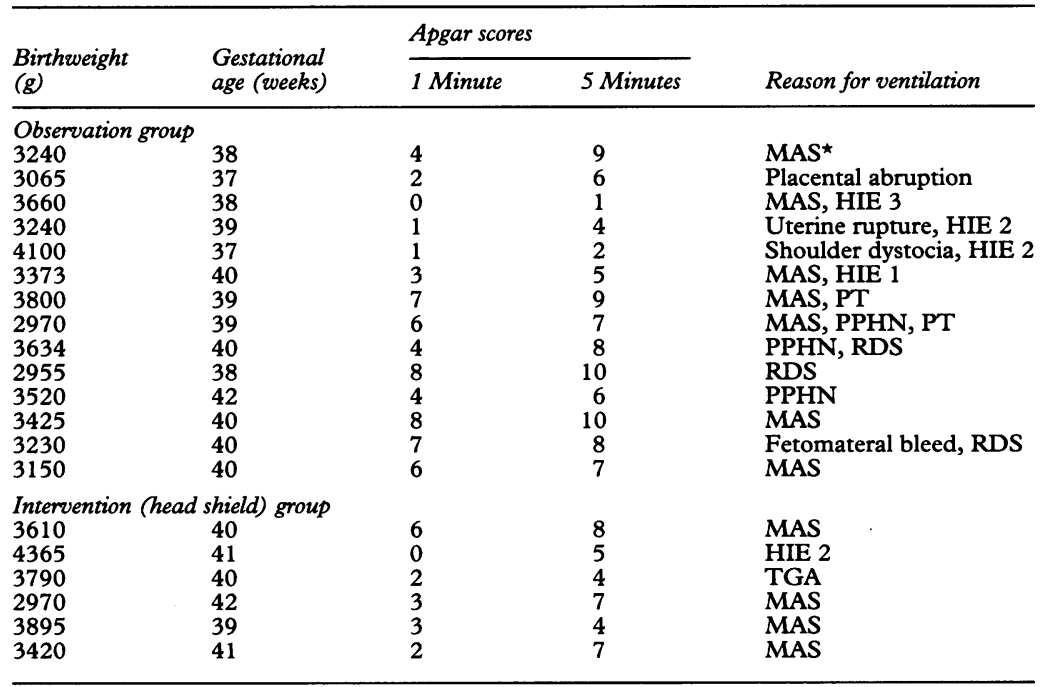

^MAS: meconium aspiration syndrome; PT: pneumothorax; PPHN: persistent pulmonary hypertension of the neonate; RDS: respiratory distress syndrome; TGA: transposition of great arteries; HIE: hypoxic-ischaemic encephalopathy. infant is reported here. Temperatures were analysed every 10 minutes from 30 minutes before the heater restarted, until 30 minutes afterwards.

\section{INTERVENTION GROUP}

In a second intervention group of six additional infants the effect on the temperature responses of a reflective heat shield placed over the head was tested. The heat shield consisted of a perspex head box usually used for delivering oxygen, covered with reflective foil. For this study the radiant heater was turned off for 30 minutes, and then switched on for 30 minutes, with and without the reflecting shield. The studies were performed in alternating order. The scalp over the fontanelle, rectal, and back against the mattress (core body) temperatures were again analysed every 10 minutes for 30 minutes, before and 30 minutes after the heater was turned on. The infants were all supine throughout this intervention study.

Ethical approval for the studies was obtained from the Auckland Area Health Board Ethics Committee. Informed consent was obtained from the parents before starting recording.

\section{STATISTICAL METHODS}

The significance of the changes in temperature was determined for use of the shield, heater activation, and between the sites of temperature, using repeated measures analysis of variance. As a significant interaction between shield, site of temperature recording, and heater activation was found, further comparisons were made using the paired $t$ test, with the significance adjusted using the Bonferroni correction for multiple comparisons. All data are presented as mean (SD), except in fig 1, where the temperatures are presented as mean (SEM).

\section{Results}

The infants were $39 \cdot 3$ ( 1.8 weeks) of gestation and weighed 3383 (331) g, with Apgar scores of 5 (3) and 7 (2) at 1 and 5 minutes, respectively; the observation and intervention group were not significantly different (table 1 ). ${ }^{1}$

In the 14 infants at the end of the (first) period, when the heater was off for at least 30 minutes, the rectal and scalp over the fontanelle temperatures were $36.8(0.3)$ and $35.6(0.3)^{\circ} \mathrm{C}$, respectively, a difference of 1.2 $(0 \cdot 2)^{\circ} \mathrm{C}$. When the radiant heater was turned on, the rectal and scalp temperatures were $37 \cdot 1$ $(0.5)$ and $36.9(0.6)^{\circ} \mathrm{C}$ after 30 minutes, a difference of only $0.2(0.5)^{\circ} \mathrm{C}(\mathrm{P}<0.001$, compared with when the heater was off). Three of the infants had scalp temperatures that were transiently higher than rectal temperature after heater activation; the greatest difference was $1.0^{\circ} \mathrm{C}, 10$ minutes after heater had been put on.

The core body, core head and rectal temperatures were not significantly different from each other at any time $(P=0.58$, repeated measures ANOVA), and showed a similar time 
sequence of changes, with a gradual fall when the heater was off and rise when it was on $(P<0.04)$. The core body temperature was $36.8(0.3)^{\circ} \mathrm{C}$ at the end of the heater off period, and $37.4(0.9)^{\circ} \mathrm{C}$ after 30 minutes with the heater on. The core head temperatures were $36.7(0.3)^{\circ} \mathrm{C}$ with the heater off and $37 \cdot 2$ $(0.5)^{\circ} \mathrm{C}$ after the heater was on: $0.07(0.06)^{\circ} \mathrm{C}$ less than rectal and $0 \cdot 1(0 \cdot 1)$ higher than rectal temperature, respectively.

\section{EFFECT OF REFLECTIVE HEAT SHIELD IN} INTERVENTION GROUP

In six infants a similar pattern of changes was found when the heater was switched off for 30 minutes, and then switched on for 30 minutes (fig 1). ${ }^{1}$ Analysis of variance suggested that there were significant effects for the site of temperature measurements (rectal vs scalp temperatures, $P<0.001$ ) and of heater activation $(P=0.005)$, with significant interactions between use of shield, heater activation, and site of measurement $(P<0.0001)$.

After 30 minutes with the heater switched off the rectal and scalp temperatures were 36.9 $(0.5)^{\circ} \mathrm{C}$ and $35.4(0.7)^{\circ} \mathrm{C}$, respectively, a rectal-scalp temperature difference of $1.5(0.4)^{\circ} \mathrm{C}$. When the heater started this gradient fell to $-0.05(0.52)^{\circ} \mathrm{C}$ after 10 minutes $(P<0.001$ vs heater off), and was $0.24(0.47)^{\circ} \mathrm{C}(\mathrm{P}<0.01)$ after 30 minutes of heating. In three of the six the scalp temperature transiently exceeded the rectal temperature, after 10 minutes of heating. In contrast, when a heat shield was placed over the head, the difference between the rectal and scalp temperatures did not change significantly when the heater was turned back on $(1.5$ $(0.5)^{\circ} \mathrm{C}$ at 0 minutes vs $1.4(0.4)^{\circ} \mathrm{C}$ at 10 minutes and $1.3(0.4)^{\circ} \mathrm{C}$ at 30 minutes; not significant).

The use of the heat shield did not significantly alter the rectal temperatures at any stage of the study, but there was, of course, a significant interaction between time and heater activation, as the rectal temperature responded gradually to the external heating $(P<0 \cdot 0001)$. Without a heat shield the rectal temperature initially fell from $37.3(0.5){ }^{\circ} \mathrm{C}$ to $36.9(0.5)^{\circ} \mathrm{C}$ in 30 minutes, then rose to $37 \cdot 3(0.5)^{\circ} \mathrm{C}$ after 30 minutes with the heater on. These changes are similar to those seen with the shield in position, when the rectal temperature fell from $37.2(0.4)$ to $36.9(0.4)$ then rose to $37 \cdot 1$ $(0.4)^{\circ} \mathrm{C}$. Very much like the observational study, the core body temperature (the back of the trunk against the mattress) of the infants was very similar to, and changed in parallel with, the rectal temperatures throughout the intervention study; the back temperatures were $0.02(0.18)^{\circ} \mathrm{C}$ and $0.08(0.16)^{\circ} \mathrm{C}$ higher, respectively (not significant) than rectal temperatures at the end of the two periods with the heater off. This difference did not vary significantly over time.

\section{Discussion}

During continuous observation of term ventilated infants under a radiant heater in the first
48 hours of life, we found that usually the rectal temperature was greater than that of the scalp over the fontanelle by a mean of $1 \cdot 2^{\circ} \mathrm{C}$. However, this gradient was abolished when the heater was activated; in some infants the scalp temperature transiently exceeded the rectal temperature, by up to $0.5^{\circ} \mathrm{C}$. Similar large swings in temperature are seen in preterm infants where the incubator temperature is servo-controlled from the abdominal skin; these have been related to thermistor attachment and to several aspects of heater design, particularly the feedback systems. ${ }^{12}$

In the intervention protocol there was a slow and modest decline in rectal temperature with the heater off and a similarly slow rise in the $\mathbf{3 0}$ minutes after the heater was turned on. The scalp temperature was $1.5(0.4)^{\circ} \mathrm{C}$ below rectal temperature in the 30 minutes when the heater was off. In contrast, the rectal to scalp temperature gradient was lost within 10 minutes of the heater starting, and was only $0 \cdot 2^{\circ} \mathrm{C}$ after 30 minutes. A head heat shield completely prevented this rapid loss of the rectal-scalp gradient. These data suggest that the acute rise in scalp temperature is due to direct infrared irradiation, rather than central warming. ${ }^{13}$

Although relatively little is known of the changes in normal human brain temperatures, limited studies in adult neurosurgical patients suggest that there is a normal positive gradient between the temperature of the lateral ventricles, and both the rectal and epidural temperatures. The lateral ventricular temperature was a mean of $0.33^{\circ} \mathrm{C}$ above that of the rectum; this gradient was much higher in a few patients (up to $2.3^{\circ} \mathrm{C}$ ), but was abolished or reversed in up to $10 \%$ of patients, at times. The lateral ventricular temperature was always greater than the epidural temperature by between 0.4 and $1.0^{\circ} \mathrm{C} .{ }^{6}$ Thus, although rectal temperature came close to epidural temperature in most patients, in a few there were noticeable discrepancies of up to $1^{\circ} \mathrm{C}$.

Clearly such invasive measurements will seldom be possible. In the present study the zero gradient method was used to follow core body and head temperatures. This methodology was first proposed by Dollberg et $a l,{ }^{10}$ as a safe, non-invasive alternative to rectal monitoring, in an experimental study of piglets. The back of trunk temperatures in the present study very closely tracked the rectal temperature, confirming that this site, insulated by the underlying mattress, provides an excellent estimate of core temperature.

Subsequently, a very similar approach has been used clinically by Simbruner et al, ${ }^{7}$ to follow the core temperature of the head in premature and term infants. They examined the importance of using measured heat flux to standardise the estimate of core temperature to zero, compared with the empirical estimate based on skin temperature measured from an insulated site. This adjustment was shown to lead to a difference of less than $0 \cdot 2^{\circ} \mathrm{C}$. These authors also directly measured the temperature gradient between the intraventricular space and skin in two premature infants. In these infants there was an initial gradual drop in 
temperature $\left(0.3-0.4^{\circ} \mathrm{C}\right)$ between the lateral ventricles to the cortex, and then about $1^{\circ} \mathrm{C}$ drop over the last $1 \mathrm{~cm}$ of cortex and skin. ${ }^{7}$ This consistent gradient within the brain clearly reflects the normal heat loss from the newborn head even in the relatively warm environment of the incubator. ${ }^{8}$ The gradient between the core temperature of the head, and body (oesophageal) temperature in the infants, was $0.72(0 \cdot 12)^{\circ} \mathrm{C} .^{7}$ This slightly larger gradient, compared with adults, might be suggested to reflect proportionately larger heat production by the head in infants, or differences in the actual site of measurements, and the effects of anaesthesia.

In the present study, however, where the infants were nursed under overhead heaters, we found no significant difference between the core head and body temperatures. This difference is probably due to the lower background environmental temperature on a heat table compared with the incubators. ${ }^{12} 13$ This will have increased radiative heat loss in these infants, reducing core head temperature. ${ }^{89}$

Heat flux from the head but not core head temperature, has been related to environmental temperature, over a limited range of temperatures in an infant incubator. ${ }^{7}$ Furthermore, the gradient was substantially increased in infants with reduced cerebral blood flow $\left(0.9-1 \cdot 2^{\circ} \mathrm{C}\right)$, and there was a trend to a reduction $\left(0.16^{\circ} \mathrm{C}\right)$ in infants with severe encephalopathy, and hence reduced cerebral metabolism. Together, these data were taken to imply that cerebral metabolism and blood flow were more important factors in regulating core head temperature than superficial radiation. The data in the present study, where scalp temperature rose sharply when the heater was on, but core head temperature remained similar to core body temperature (and rectal temperature), support such a key role for cerebral blood flow in regulating infants' core head temperature. Nevertheless, a rise in scalp temperature, flattening the heat gradient between brain and scalp, must be accompanied by a rise in the temperature of intermediate or superficial brain structures - that is, the final few centimetres of the brain under the scalp, described above. This gradient sems to be normal in term infants; the effect of obliterating it is unknown, but might be adverse in infants with hypoxic-ischaemic encephalopathy.

Relative hyperthermia of even one to two degrees during or shortly after an ischaemic insult has similar effects to a prolongation of the period of ischaemia, and, is suggested, could promote cortical pannecrosis in animal studies. ${ }^{2}$ The mechanism of this adverse influence of mild hyperthermia is unclear but may be related to a dramatic increase in the release of excitotoxins. ${ }^{14}$ Preventing even a transient rise in brain temperature after normothermic cerebral ischaemia may reduce neuronal loss. ${ }^{3}$ Although only superficial cortical structures are likely to be affected by the rapid rise of the scalp temperature seen in the present study, watershed (parietal parasagittal) and middle cerebral artery damage of cortical regions are common patterns of injury in the asphyxiated term newborn. ${ }^{15-18}$ Further study of the significance of the effect of radiant heating on the cerebral temperature gradient is needed.

In conclusion, infants nursed under a radiant heater may have rapid increases in the temperature of the scalp during standard care, with loss of the head core to scalp gradient, but no change in the core head temperature relative to body temperature. A reflecting shield over the head of the infant prevents this superficial heating.

This study was supported in part by a grant from the Health This study was supported in part
Research Council of New Zealand.

1 Ginsberg MD, Sternau LL, Globus MY, Dietrich WD, Busto $R$. Therapeutic modulation of brain temperature: relevance to ischemic brain injury. Cerebrovasc Brain Metab Rev 1992; 4: 189-225.

2 Dietrich WD, Busto R, Valdes I, Loor Y. Effects of normothermic versus mild hyperthermic forebrain ischemia in rats. Stroke 1990; 21: 1318-25.

3 Kuroiwa T, Bonnekoh P, Hossmann KA. Prevention of postischemic hyperthermia prevents ischemic injury of CAl neurons in gerbils. 7 Cereb Blood Flow Metab 1990 10: 550-6.

4 Stratton D. Aural temperature of the newborn infant. Arch Dis Child 1977; 52: 865-9.

5 Mellergard P, Nordstrom CH. Intracerebral temperature in neurosurgical patients. Neurosurg 1991; 28: 709-13.

6 Mellergard P, Nordstrom $\mathrm{CH}$. Epidural temperature and possible intracerebral temperature gradients in man. $B r \mathcal{F}$ Neurosurg 1990; 4: 31-8.

7 Simbruner G, Nanz S, Fleischhacker E, Derganc M. Brain temperature discriminates between neonates with damaged, hypoperfused, and normal brains. Am $\mathcal{f}$ Perinato 1994; 11: 137-43.

8 Weninger M, Simbruner G, Malamitsi Puchner A. Heat flux from the head surface in healthy newborns and in newborns with cerebral pathology. Wien Klin Wochenschr 1989; 101: 529-33.

9 Karlsson H, Hanel S-E, Nilsson $K$, Olegard $R$. Measurement of skin temperature and heat flow from skin in term newborn babies. Acta Paediatr 1995; 84: 605-12.

10 Dollberg S, Xi Y, Donnelly MM. A noninvasive transcutaneous alternative to rectal thermometry for continuous measurement of core temperature in the piglet. Ped Res 1993; 34: 512-17.

11 Gluckman PD, Gunn TR, Johnston BM, Quinn JP. Manipulation of the temperature of the fetal lamb in utero. In: Nathanielsz P, ed. Animal Models in Fetal Physiology III. New York: Perinatology Press, 1984: $37-56$.

12 LeBlanc $\mathrm{MH}$. Thermoregulation: incubators, radiant warmers, artificial skins, and body hoods. Clin Perinatol 1991; 18: 403-22.

13 Sinclair A. The measurement of radiant temperature in neonatal thermal environments. Biomed Instrum Technol 1992; 26: 400-7.

14 Takagi K, Ginsberg MD, Globus MYT, Martinez E, Busto $R$. Effect of hyperthermia on glutamate release in ischemic penumbra after middle cerebral artery occlusion in rats. Am $\mathcal{J}$ Physiol-Heart Circ Phys 1994; 36: H1770-6.

15 Volpe J, Herscovitch P, Perlman JM, Kreusser K, Raichle ME. Positron emission tomography in the asphyxiated ME. Positron emission tomography in the asphyxiated term newborn: parasagittal impairm

16 Barmada MA, Moossy J, Shuman RM. Cerebral infarcts with arterial occlusion in neonates. Ann Neurol 1979; 6: 45-52.

17 Barkovich AJ, Truwit CL. Brain damage from perinatal asphyxia: correlation of MR findings with gestational age. Am $\mathcal{f}$ Neuroradiol 1990; 11: 1087-96.

18 Pasternak JF, Predey TA, Mikhael MA. Neonatal asphyxia: vulnerability of basal ganglia, thalamus, and brainstem. Pediatr Neurol 1991; 7: 147-9. 